\title{
Untersuchungen äber die Sexualität und Entwicklung von marinen Algen
}

Von Björn Föyn, Berlin-Dahlem

Seitdem Professor Hartmann den Nachweis der Getrenntgeschlechtlichkeit und der im Rahmen derselben auftretenden relativen Sexualität von Ectocarpus siliculosus von Neapel erbracht hatte, werden von Prof. Hartmann und seinen Assistenten Dr. Hämmerling und Föyn mehrere andere marine Algen untersucht. Von diesen Untersuchungen sind die an Ulva und Enteromorpha sowie an Cladophora und Chaetomorpha jetzt so. weit gediehen, daß sie ein einigermaßen klares Urteil erlauben. Sämtliche Gattungen sind isogam. Es gibt zoosporenbildende Pflanzen und gametenbildende Pflanzen. Die Zoosporen sind viergeißelig und größer als die Gameten, die zwei Geißeln haben. Bei dèr Herstellung zahlreicher Kombinationen zwischen den Gameten der verschiedenen Pflanzen ergab sich, daß bei den Arten Vlva lactuca $L$., Enteromorpha compressa (L.) Grev., Ent. ramulosa (Engl. Bot.) Hook, Cladophora pellucida (Huds.) Kütz, Clad. utriculosa Kütz, Clad. Neesiorum Ag., Chaetomorpha linum (Fl. Dan.) Kütz und Chaet. aerea (Dillw.) $\mathrm{Kütz}$ strenge Getrenntgeschlechtlichkeit vorliegt. Bei Enteromorpha wurde auch ein Fall vor relativer Sexualität beobachtet. Die zytologische Untersuchung von Vlva, Cladophora und Chaetomorpha ergab, daß die zoosporenbildenden Pflanzen diploid sind, während die gametenbildenden Pflanzen haploid sind, und daß die Reduktionsteilung in den Zoosporangien vor sich geht. Es liegt somit bei diesen Gattungen antithetischer Generationswechsel vor. Ausführliche Berichte über diese Untersuchungen erscheinen in den „Berichten der Deutschen Botanischen Gesellschaft" Band 47, Heft 7, S. 485 und 495.

\section{Multipolare Sexualität bei Brandpilzen}

\author{
Von R. Banch, Rostock
}

In einer etwa 6 Jahre zurïckliegenden Veröffentlichung hatte der Vortragende den auffallenden Befund mitgeteilt, daß bei dem getrenntgeschlechtlichen Brandpilz Ustilago longissima mehr als zwei Geschlechter vorkommen. Er hatte damals drei Geschlechter gefunden, die als A, B und $\mathbf{C}$ - der Pilz ist streng isogam - bezeichnet wurden. Auch bei einer weiteren Bearbeitung dieses Befundes durch H. Kämmerling (s. Zeitschr. f. Bot. Bd. 22, 1929) wurden immer nur drei Geschlechter gefunden. Bei der Durchprüfung des Pilzes von ungarischen Fundorten dagegen konnte der Vortragende ganz andere Verhältnisse nachweisen. Innerhalb eines jeden Standortes wurden vier verschiedene Geschlechter gefunden, von denen jedes nur mit einem der anderen geschlechtlich reagierte. Die Befunde entsprechen 